\title{
Excited State Properties of 7-hydroxy-4-methylcoumarin in the Gas Phase and in Solution. A Theoretical Study
}

\author{
I. Georgieva, N. Trendafilova \\ Institute of General and Inorganic Chemistry, Bulgarian Academy of Sciences, Sofia, \\ Bulgaria \\ A. Aquino, H. Lischka \\ Institute for Theoretical Chemistry, Währingerstraße 17, University of Vienna, A-1090 \\ Vienna, Austria
}

\section{TDDFT/B3LYP/SVPD results}

Optimized Cartesian geometries $(\AA)$, total energies in Hartree

\section{Enol form in the ground state $\left(\mathrm{C}_{\mathrm{s}}\right.$ symmetry $)$}

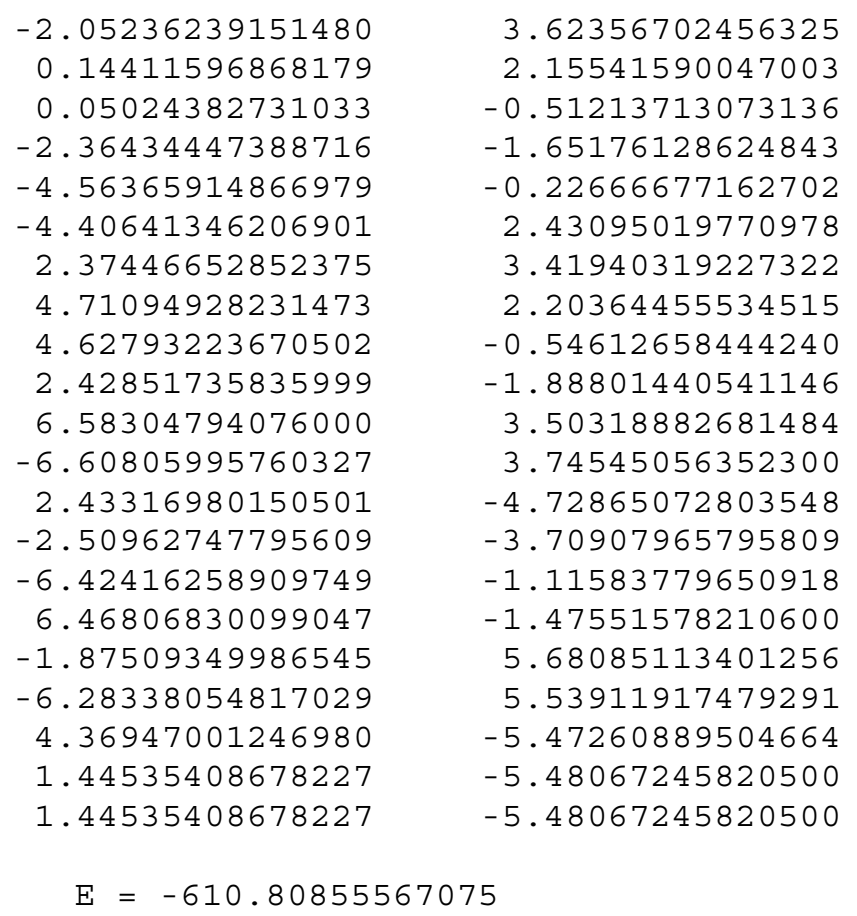

\section{Enol form in ${ }^{1} \pi \pi^{*}$ excited state $\left(C_{s}\right.$ symmetry $)$}

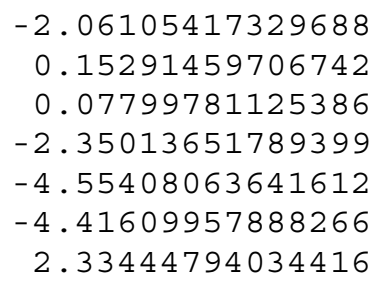

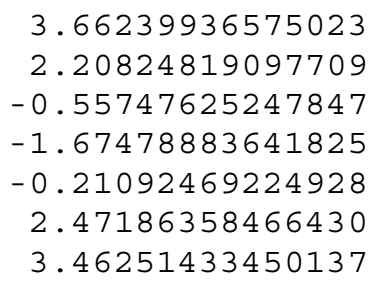

$\begin{array}{ll}0.00000000000000 & \mathrm{C} \\ 0.00000000000000 & \mathrm{C} \\ 0.00000000000000 & \mathrm{C} \\ 0.00000000000000 & \mathrm{C} \\ 0.00000000000000 & \mathrm{C} \\ 0.00000000000000 & \mathrm{C} \\ 0.00000000000000 & \mathrm{O} \\ 0.00000000000000 & \mathrm{C} \\ 0.00000000000000 & \mathrm{C} \\ 0.00000000000000 & \mathrm{C} \\ 0.00000000000000 & \mathrm{O} \\ 0.00000000000000 & \mathrm{O} \\ 0.00000000000000 & \mathrm{C} \\ 0.00000000000000 & \mathrm{H} \\ 0.00000000000000 & \mathrm{H} \\ 0.00000000000000 & \mathrm{H} \\ 0.00000000000000 & \mathrm{H} \\ 0.00000000000000 & \mathrm{H} \\ 0.00000000000000 & \mathrm{H} \\ 1.67244251201679 & \mathrm{H} \\ -1.67244251201679 & \mathrm{H}\end{array}$

0.00000000000000

C

0.00000000000000

0.00000000000000

0.00000000000000

0.00000000000000

0.00000000000000

0.00000000000000

$$
\text { C }
$$$$
\text { (1) }
$$$$
\text { C }
$$$$
\text { C }
$$$$
\text { C }
$$$$
0
$$$$
\text { C }
$$$$
\mathrm{H}
$$$$
\mathrm{H}
$$$$
\mathrm{H}
$$
H 

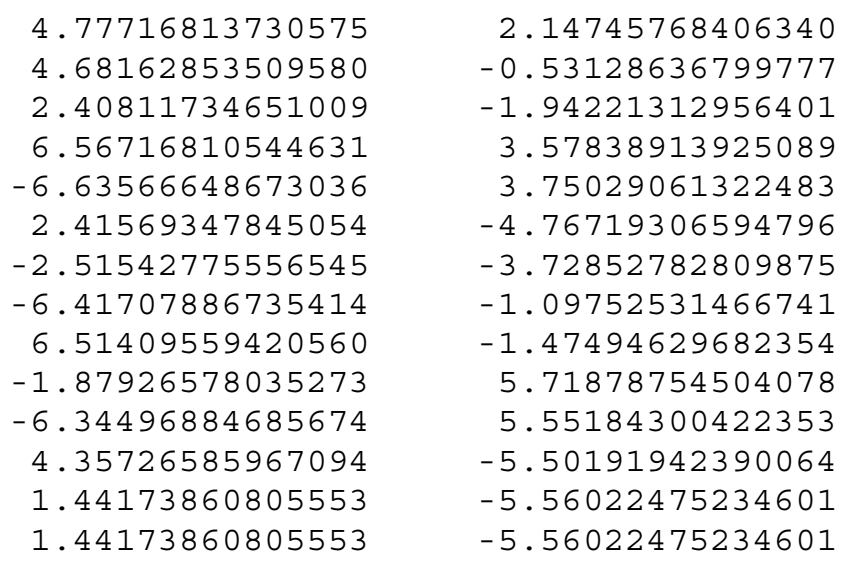

0.00000000000000

0.00000000000000

0.00000000000000

0.00000000000000

0.00000000000000

0.00000000000000

0.00000000000000

0.00000000000000

0.00000000000000

0.00000000000000

0.00000000000000

0.00000000000000

1.67275661277950

$-1.67275661277950$

$\mathrm{C}$
$\mathrm{C}$
$\mathrm{C}$
$\mathrm{O}$
$\mathrm{O}$
$\mathrm{C}$
$\mathrm{H}$
$\mathrm{H}$
$\mathrm{H}$
$\mathrm{H}$
$\mathrm{H}$
$\mathrm{H}$
$\mathrm{H}$
$\mathrm{H}$

$E=-610.6640966081611$

\section{Enol form in ${ }^{1} \pi \pi^{*}$ excited state ( $C_{1}$ symmetry)}

-2.04213094635218
0.15182625962366
0.06842586610140
-2.34547247136554
-4.54429664231408
-4.39567291959375
2.29925702238847
4.74668289965977
4.65466296665695
2.42038303824646
6.40894039102057
-6.60657944182046
2.44182067263845
-2.53347082150669
-6.40710448924151
6.46380191971627
-1.83788566859913
-6.30407732833841
4.38675096127785
1.45766979830665
1.49113959400838

$$
\begin{array}{r}
3.66002249548255 \\
2.17393612413991 \\
-0.57981415918645 \\
-1.66688922112780 \\
-0.17911999011162 \\
2.49727529234985 \\
3.35383583052191 \\
2.13316972422523 \\
-0.52348637600828 \\
-1.95842599066692 \\
3.67175863156249 \\
3.79010503408027 \\
-4.78301300372280 \\
-3.71908109353249 \\
-1.05854527367451 \\
-1.44537021274274 \\
5.71327077938533 \\
5.58871752196562 \\
-5.50976654912653 \\
-5.62533799005200 \\
-5.53994256650729
\end{array}
$$

$E=-610.6645335508607$

$\begin{array}{rl}0.08631977561696 & \mathrm{C} \\ 0.34050565562284 & \mathrm{C} \\ 0.19836272551160 & \mathrm{C} \\ 0.00806797503719 & \mathrm{C} \\ -0.11862562694859 & \mathrm{C} \\ -0.10542291406053 & \mathrm{C} \\ 0.85784903159838 & \mathrm{O} \\ 0.04176518716601 & \mathrm{C} \\ -0.16148402014301 & \mathrm{C} \\ 0.14373225498555 & \mathrm{C} \\ -0.28361640329369 & \mathrm{O} \\ -0.25970894227953 & \mathrm{O} \\ 0.05924392220063 & \mathrm{C} \\ -0.05107661545251 & \mathrm{~h} \\ -0.24198811472300 & \mathrm{~h} \\ -0.52665381216153 & \mathrm{~h} \\ 0.16127800865199 & \mathrm{~h} \\ -0.25966112575815 & \mathrm{~h} \\ 0.05793244108443 & \mathrm{~h} \\ 1.69812273012924 & \mathrm{~h} \\ -1.64674074459394 & \mathrm{~h}\end{array}$$$
\text { ○ }
$$

$\bigcirc$

C$$
\mathrm{h}
$$

h

h h

\section{Enol form in ${ }^{1} n \pi^{*}$ excited state $\left(C_{s}\right.$ symmetry $)$}
$-2.02711203575803$
0.12169075936939
0.06769619405976
$-2.39105607687259$
$-4.57075750953985$
$-4.41236383754180$
2. 41210805546305
4.57406351434779
4.68632667333458
2. 38448965983981
6.52603815504498

\author{
3. 61864458793210 \\ 2. 12265288666437 \\ $-0.56263267682915$ \\ $-1.66814723844641$ \\ $-0.20150718786723$ \\ 2. 44413099866963 \\ 3. 40003811981003 \\ 2. 09321632491364 \\ $-0.48840867342629$ \\ $-1.91812103492233$ \\ 3. 66082757190952
}
0.00000000000000
0.00000000000000
0.00000000000000
0.00000000000000
0.00000000000000
0.00000000000000
0.00000000000000
0.00000000000000
0.00000000000000
0.00000000000000
0.00000000000000 
$-6.60978822942320$

2. 43975670692013

$-2.56003825440516$

$-6.43802415546673$

6.52916155737569

$-1.82552431223397$

$-6.26635730978741$

4. 38813662167755

1. 47293101037413

1. 47293101037413
3.79010731943273

$-4.74664277407525$

$-3.72294715493620$

$-1.07933489598702$

$-1.40273884604858$

5.67347267154934

5.57887818185688

$-5.46413469698256$

$-5.54539704536449$

$-5.54539704536449$
0.00000000000000

0.00000000000000

0.00000000000000

0.00000000000000

0.00000000000000

0.00000000000000

0.00000000000000

0.00000000000000

1. 67180750828363

$-1.67180750828363$
0

C

$\mathrm{H}$

$\mathrm{H}$

$\mathrm{H}$

$\mathrm{H}$

$\mathrm{H}$

$\mathrm{H}$

$\mathrm{H}$

$\mathrm{H}$

$E=-610.6667524762279$

\section{Keto form in the ground state $\left(\mathrm{C}_{\mathrm{s}}\right.$ symmetry $)$}

$-2.56862419970370$

$-0.47360414947484$

$-0.52682174301692$

$-3.01105287814802$

$-5.14807381721534$

$-5.08875682035058$

1. 87450492512572

4. 01150214886768

4. 06653769145579

1. 73490300969489

1. 80036360088928

6.07165025655721

$-7.04431100900106$

$-3.15589652674594$

$-7.01905297055939$

5.88939167382976

$-2.41454333082487$

3. 75101440304185

0.83508763680038

0.83508763680038

5.58658388428626
3.69073003232548

2. 19914662982222

$-0.53385410235464$

$-1.65601405129479$

- 0.22555761756399

2. 56234524237997

3. 38563620899607

2. 06155318052953

$-0.52247277414759$

$-1.87839817571479$

$-4.72251991696794$

3. 50187886941348

3. 83877716902860

$-3.71522602274383$

$-1.09779055828784$

$-1.47695830633154$

5.74610795120028

$-5.42796495611725$

$-5.50051358260436$

$-5.50051358260436$

5.26749677708900
0.00000000000000

0.00000000000000

0.00000000000000

0.00000000000000

0.00000000000000

0.00000000000000

0.00000000000000

0.00000000000000

0.00000000000000

0.00000000000000

0.00000000000000

0.00000000000000

0.00000000000000

0.00000000000000

0.00000000000000

0.00000000000000

0.00000000000000

0.00000000000000

1. 67212049317747

$-1.67212049317747$

0.00000000000000
C

C

C

C

C

C

$\mathrm{O}$

C

$\mathrm{C}$

C

O

O

$\mathrm{H}$

$\mathrm{H}$

$\mathrm{H}$

$\mathrm{H}$

$\mathrm{H}$

$\mathrm{H}$

$\mathrm{H}$

$\mathrm{H}$

$E=-610.76898344087$

\section{Keto form in ${ }^{1} \pi \pi^{*}$ excited state $\left(\mathrm{C}_{\mathrm{s}}\right.$ symmetry $)$}

$-2.60773158085753$

$-0.42589742053908$

$-0.53952615650535$

$-3.01206086672520$

$-5.20430801476294$

$-5.13747292746311$

1. 80124370019757

3. 99869713291585

4.08259425321026

1. 88139376861175

1. 83429356724289

6.06704055332408

$-7.03303693094733$

$-3.16414192917341$

$-7.05651308507158$
3. 67168926274287

2.11627595548048

$-0.52124420913393$

$-1.60222236733718$

$-0.19124443112562$

2. 55481191601033

3. 37543325936664

2. 10163137895793

$-0.55820243471995$

$-1.94330679245237$

$-4.75409854216074$

3. 56056360582225

3. 95595153183514

$-3.66281452867922$

$-1.09619367479020$
0.00000000000000

0.00000000000000

0.00000000000000

0.00000000000000

0.00000000000000

0.00000000000000

0.00000000000000

0.00000000000000

0.00000000000000

0.00000000000000

0.00000000000000

0.00000000000000

0.00000000000000

0.00000000000000

0.00000000000000
C

C

C

C

C

C

O

C

C

C

C

O

O

$\mathrm{H}$

$\mathrm{H}$ 
5. 94245100723532

$-2.42196322651197$

3. 74551450181811

0.80252262326486

0.80252262326486

5.60216938084790
$-1.44674230245640$

5.72489765068702

$-5.56207397218576$

$-5.48620156459216$

$-5.48620156459216$

5.32850339007186
0.00000000000000

0.00000000000000

0.00000000000000

1. 66574303418544

$-1.66574303418544$

0.00000000000000
$\mathrm{H}$

$\mathrm{H}$

$\mathrm{H}$

$\mathrm{H}$

$\mathrm{H}$

$\mathrm{H}$

$E=-610.6696516924322$

\section{Keto form in ${ }^{1} n \pi^{*}$ excited state $\left(C_{s}\right.$ symmetry $)$}

$-2.59688587745463$

$-0.46142859694637$

$-0.54057073714012$

$-2.97998403841117$

$-5.17380188501187$

$-5.01010677514706$

1. 82526583692744

4.00230773134605

4. 06211336802184

1. 80921057937654

1. 80920976084604

6.06423048874784

$-7.03897462203874$

$-3.17455439301810$

$-7.03535691166593$

5.90848269016497

$-2.40992513101294$

3. 74207727922647

0.81914243313593

0.81914243313593

5. 56839468170745

\author{
3.67004318595563 \\ 2. 13919451025149 \\ $-0.52827306335722$ \\ $-1.60076853418969$ \\ - 0.14391945791156 \\ 2. 52813522130882 \\ 3. 39582627383021 \\ 2. 06330170379435 \\ $-0.53535081475162$ \\ $-1.93655514084411$ \\ $-4.76003883853630$ \\ 3. 52997896737131 \\ 3. 94171857202255 \\ $-3.65490754382921$ \\ - 1.02521356564778 \\ $-1.45034842562622$ \\ 5.72025466460146 \\ $-5.51730161730104$ \\ $-5.53478668295110$ \\ $-5.53478668295110$ \\ 5. 28900115161925
}

0.00000000000000

0.00000000000000

0.00000000000000

0.00000000000000

0.00000000000000

0.00000000000000

0.00000000000000

0.00000000000000

0.00000000000000

0.00000000000000

0.00000000000000

0.00000000000000

0.00000000000000

0.00000000000000

0.00000000000000

0.00000000000000

0.00000000000000

0.00000000000000

1. 66914542646295

$-1.66914542646295$

0.00000000000000
C

C

C

C

C

C

$\mathrm{O}$

C

C

C

C

O

O

$\mathrm{H}$

$\mathrm{H}$

$\mathrm{H}$

$\mathrm{H}$

$\mathrm{H}$

$\mathrm{H}$

$\mathrm{H}$

$\mathrm{H}$

$E=-610.6770805408733$

\section{Cationic form in the ground state $\left(C_{s}\right.$ symmetry $)$}

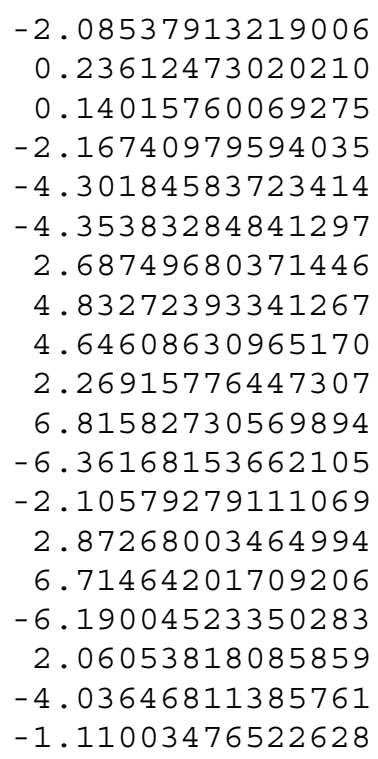

\author{
2.15803936431081 \\ 0.76793578804268 \\ $-1.90975774131353$ \\ $-3.10709001259622$ \\ - 1.81611038161287 \\ 0.81387490051517 \\ 1. 87589754021634 \\ 0.40478151710001 \\ $-2.27302180598658$ \\ $-3.44346125612441$ \\ $-3.55534121281081$ \\ $-3.17430817828088$ \\ 4. 98243056324923 \\ 3. 92858092788571 \\ 1. 24822676890430 \\ 1. 74528315963686 \\ $-5.49561320986786$ \\ 5.73602692546023 \\ 5. 72703773048078
}

$\begin{array}{ll}0.00000000000000 & \mathrm{C} \\ 0.00000000000000 & \mathrm{C} \\ 0.00000000000000 & \mathrm{C} \\ 0.00000000000000 & \mathrm{O} \\ 0.00000000000000 & \mathrm{C} \\ 0.00000000000000 & \mathrm{C} \\ 0.00000000000000 & \mathrm{C} \\ 0.00000000000000 & \mathrm{C} \\ 0.00000000000000 & \mathrm{C} \\ 0.00000000000000 & \mathrm{C} \\ 0.00000000000000 & \mathrm{O} \\ 0.00000000000000 & \mathrm{O} \\ 0.00000000000000 & \mathrm{C} \\ 0.00000000000000 & \mathrm{H} \\ 0.00000000000000 & \mathrm{H} \\ 0.00000000000000 & \mathrm{H} \\ 0.00000000000000 & \mathrm{H} \\ 0.00000000000000 & \mathrm{H} \\ 1.67242201866671 & \mathrm{H}\end{array}$
C

C

O

C

C

C

C

o

O

C

H

$\mathrm{H}$

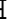

H

$\mathrm{H}$ 
-1.11003476522628
6.56213722440103
-6.01310220734646

5.72703773048078

$-5.36446106172467$

$-4.97801429019417$
-1.67242201866671
0.00000000000000
0.00000000000000

$\mathrm{H}$

$\mathrm{H}$

$\mathrm{H}$

$E=-611.16527305925$

\section{Cationic form in ${ }^{1} \pi \pi^{*}$ excited state $\left(\mathrm{C}_{\mathrm{s}}\right.$ symmetry $)$}

\begin{abstract}
$-2.18220565$
0.20610411

0.06842848

$-2.13655892$

$-4.35182889$

$-4.38439500$

2. 69721225

4.91585733

4.74742166

2.28994065

6. 84294412

$-6.41018985$

$-2.10981567$

2. 86655847

6.76816076

$-6.24136860$

2. 07205156

$-4.02427123$

$-1.10786118$

$-1.10786118$

6.54685210

$-6.02680041$
\end{abstract}

\begin{abstract}
2. 24057970
0.73926762

$-1.85767986$

$-3.15794927$

$-1.81458667$

0.82138338

1. 80724492

0.37940643

$-2.24984849$

$-3.40808010$

$-3.63248666$

$-3.23108551$

5.05160976

3.86470736

1. 28296416

1. 71962337

$-5.46194069$

5.85065791

5.80509966

5.80509966

$-5.43918980$

$-5.02106920$
\end{abstract}

$\begin{array}{rr}0.00000000 & \mathrm{C} \\ 0.00000000 & \mathrm{C} \\ 0.00000000 & \mathrm{C} \\ 0.00000000 & \mathrm{O} \\ 0.00000000 & \mathrm{C} \\ 0.00000000 & \mathrm{C} \\ 0.00000000 & \mathrm{C} \\ 0.00000000 & \mathrm{C} \\ 0.00000000 & \mathrm{C} \\ 0.00000000 & \mathrm{C} \\ 0.00000000 & \mathrm{O} \\ 0.00000000 & \mathrm{O} \\ 0.00000000 & \mathrm{C} \\ 0.00000000 & \mathrm{H} \\ 0.00000000 & \mathrm{H} \\ 0.00000000 & \mathrm{H} \\ 0.00000000 & \mathrm{H} \\ 0.00000000 & \mathrm{H} \\ 1.67337333 & \mathrm{H} \\ -1.67337333 & \mathrm{H} \\ 0.00000000 & \mathrm{H} \\ 0.00000000 & \mathrm{H}\end{array}$

$E=-611.0419656391781$

\section{Cationic form in ${ }^{1} n \pi^{*}$ excited state $\left(C_{s}\right.$ symmetry $)$}

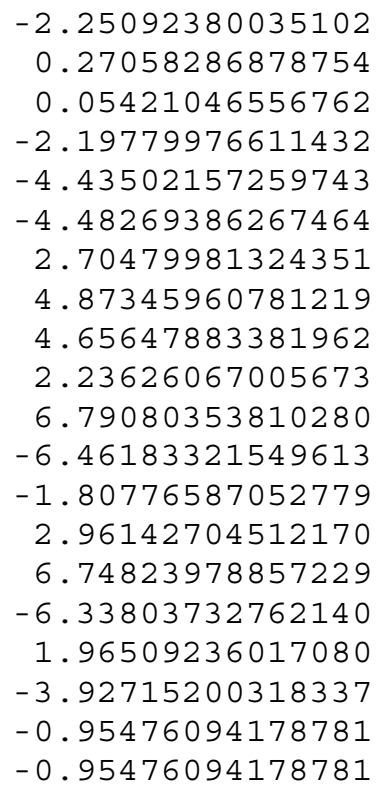

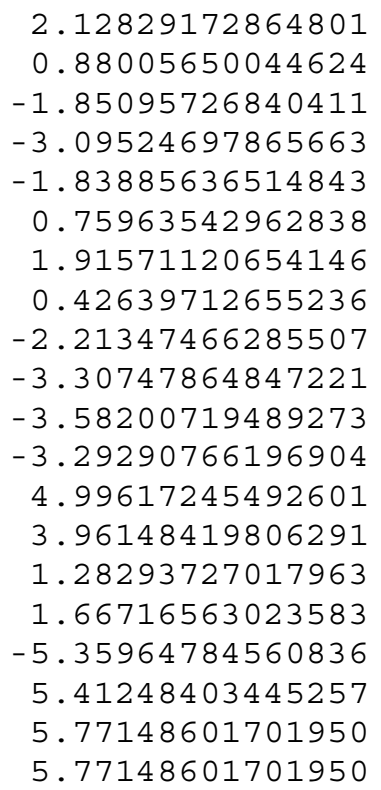
0.00000000000000
0.00000000000000
0.00000000000000
0.00000000000000
0.00000000000000
0.00000000000000
0.00000000000000
0.00000000000000
0.00000000000000
0.00000000000000
0.00000000000000
0.00000000000000
0.00000000000000
0.00000000000000
0.00000000000000
0.00000000000000
0.00000000000000
0.00000000000000
1. 72393325560333
$-1.72393325560333$ 

6.47823241635098
-6.03043987885111
$-5.38035940631648$
0.00000000000000
$-5.07333415810525$
0.00000000000000
$E=-610.9556926238123$

$\mathrm{H}$

$\mathrm{H}$

\section{Anionic form in the ground state $\left(C_{s}\right.$ symmetry $)$}

\begin{abstract}
$-2.06513868827484$
0.25816404091087

0.16843716931925

$-2.13545781315286$

$-4.43786900728752$

$-4.33050219428864$

2. 71961471622588

4.88298053126665

4. 82695727806312

2. 31195367782717

6.81195113852770

$-6.34452224896271$

$-2.07586451586176$

2. 88013248864184

6.75071392271052

$-6.14594057440522$

2. 12780033957113

$-4.01794099350469$

$-1.09139951348513$

$-1.09139951348513$
\end{abstract}

\begin{abstract}
1.61389763794236
0.25718564562475

$-2.44521242335119$

$-3.65672544271358$

$-2.41099526695606$

0.30933406864141

1. 36266593284823

$-0.07445914791349$

$-2.84010585815107$

$-3.93067638388311$

$-4.14084359163794$

$-3.71434236692586$

4.46643628145227

3. 42527768440638

0.81277263532111

1. 28652673335771

$-5.98524599891387$

5.20391569645079

5. 22848651403300

5. 22848651403300
\end{abstract}

0.00000000000000

0.00000000000000

0.00000000000000

0.00000000000000

0.00000000000000

0.00000000000000

0.00000000000000

0.00000000000000

0.00000000000000

0.00000000000000

0.00000000000000

0.00000000000000

0.00000000000000

0.00000000000000

0.00000000000000

0.00000000000000

0.00000000000000

0.00000000000000

1. 67087405842051

$-1.67087405842051$
C

$\mathrm{C}$

C

$\mathrm{O}$

C

C

C

C

C

C

O

$\mathrm{O}$

C

$\mathrm{H}$

$\mathrm{H}$

$\mathrm{H}$

$\mathrm{H}$

$\mathrm{H}$

$\mathrm{H}$

$\mathrm{H}$

$$
E=-610.27528631867
$$

\section{Anionic form in ${ }^{1} \pi \pi^{*}$ excited state $\left(\mathrm{C}_{\mathrm{s}}\right.$ symmetry $)$}

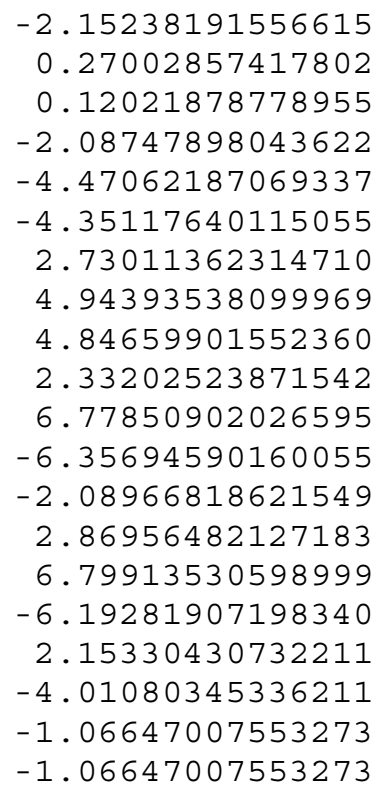

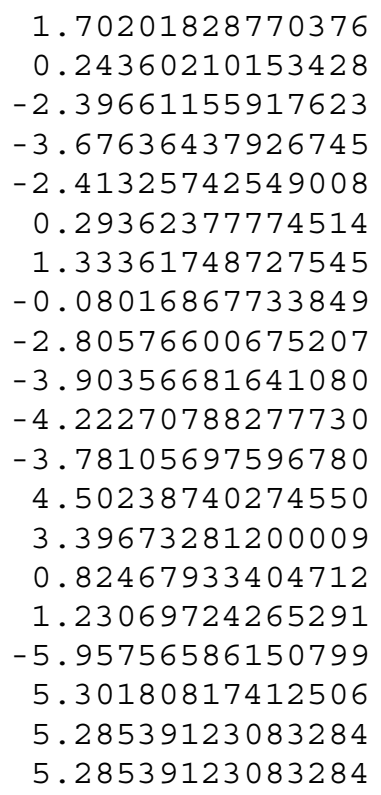

$\begin{array}{ll}0.00000000000000 & \mathrm{C} \\ 0.00000000000000 & \mathrm{C} \\ 0.00000000000000 & \mathrm{C} \\ 0.00000000000000 & \mathrm{O} \\ 0.00000000000000 & \mathrm{C} \\ 0.00000000000000 & \mathrm{C} \\ 0.00000000000000 & \mathrm{C} \\ 0.00000000000000 & \mathrm{C} \\ 0.00000000000000 & \mathrm{C} \\ 0.00000000000000 & \mathrm{C} \\ 0.00000000000000 & \mathrm{O} \\ 0.00000000000000 & \mathrm{O} \\ 0.00000000000000 & \mathrm{C} \\ 0.00000000000000 & \mathrm{H} \\ 0.00000000000000 & \mathrm{H} \\ 0.00000000000000 & \mathrm{H} \\ 0.00000000000000 & \mathrm{H} \\ 0.00000000000000 & \mathrm{H} \\ 1.66903305130950 & \mathrm{H} \\ -1.66903305130950 & \mathrm{H}\end{array}$
C C C C . . , . . . $\mathrm{H}$ H . 


\section{Anionic form in ${ }^{1} \pi \sigma^{*}$ excited state $\left(\mathrm{C}_{\mathrm{s}}\right.$ symmetry $)$}

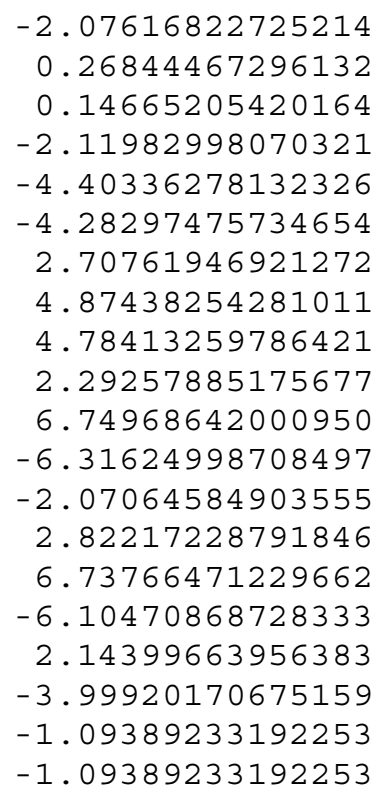

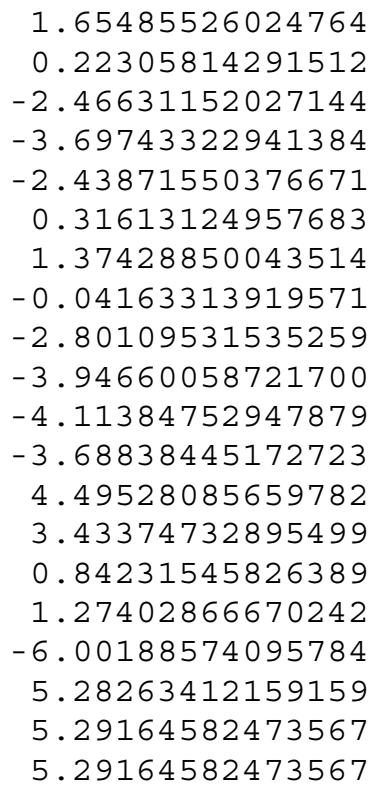

$\begin{array}{ll}0.00000000000000 & C \\ 0.00000000000000 & C \\ 0.00000000000000 & C \\ 0.00000000000000 & \text { O } \\ 0.00000000000000 & C \\ 0.00000000000000 & C \\ 0.00000000000000 & C \\ 0.00000000000000 & C \\ 0.00000000000000 & \text { C } \\ 0.00000000000000 & \mathrm{C} \\ 0.00000000000000 & \mathrm{O} \\ 0.00000000000000 & \mathrm{O} \\ 0.00000000000000 & \mathrm{C} \\ 0.00000000000000 & \mathrm{H} \\ 0.00000000000000 & \mathrm{H} \\ 0.00000000000000 & \mathrm{H} \\ 0.00000000000000 & \mathrm{H} \\ 0.00000000000000 & \mathrm{H} \\ 1.67335291458818 & \mathrm{H} \\ -1.67335291458818 & \mathrm{H}\end{array}$

$E=-610.1647985694701$

\section{$\mathrm{E}-\left(\mathrm{H}_{2} \mathrm{O}\right)_{2}$ cluster in the ground state $\left(\mathrm{C}_{\mathrm{s}}\right.$ symmetry $)$}

\begin{abstract}
$-2.56671129426699$
$-0.40879629657242$

$-0.54406776673456$

$-2.98463496395050$

$-5.15141829166034$

$-4.95708434057766$

1.85698957988812

4.13848172210227

4.03453384229651

1.80429827905405

1.76363669720329

6.04507393128873

$-7.10832812502545$

$-6.37098473479490$

10.36780484670146

$-3.16762505985348$

$-7.02840294668858$

5.86030168833898

$-2.34967013588392$

$-6.76826813095052$

3.68725579923626

0.76104914477750

0.76104914477750

$-4.87391048130609$

$-7.78254911355536$

9.07922354163800

11.96230024300013
\end{abstract}

\begin{abstract}
2.48801931279603
0.97246734956154

$-1.69151810246174$

$-2.78207482624193$

$-1.31140878489209$

1. 35286858018668

2. 19178273727430

0.93224412392342

$-1.80641102567006$

$-3.10704618624619$

$-5.94633083997248$

2. 21857178652442

2. 70729230019654

7.98279314900615

$-0.89775895794961$

$-4.83684666710667$

$-2.16625121481103$

$-2.76496920863734$

4.53643522157054

4.52072343619733

$-6.72109813431656$

$-6.67961765332204$

$-6.67961765332204$

9.01979795848391

9.13380383695356

0.42145409057095

$-0.02501161115964$
\end{abstract}

$\begin{array}{cc}0.00000000000000 & \text { C } \\ 0.0000000000000 & \mathrm{C} \\ 0.00000000000000 & \mathrm{C} \\ 0.00000000000000 & \mathrm{C} \\ 0.0000000000000 & \mathrm{C} \\ 0.00000000000000 & \mathrm{C} \\ 0.00000000000000 & \mathrm{O} \\ 0.0000000000000 & \mathrm{C} \\ 0.00000000000000 & \mathrm{C} \\ 0.00000000000000 & \mathrm{C} \\ 0.0000000000000 & \mathrm{C} \\ 0.00000000000000 & \mathrm{O} \\ 0.00000000000000 & \mathrm{O} \\ 0.00000000000000 & \mathrm{O} \\ 0.00000000000000 & \mathrm{O} \\ 0.00000000000000 & \mathrm{H} \\ 0.00000000000000 & \mathrm{H} \\ 0.00000000000000 & \mathrm{H} \\ 0.00000000000000 & \mathrm{H} \\ 0.00000000000000 & \mathrm{H} \\ 0.00000000000000 & \mathrm{H} \\ 1.67200797572498 & \mathrm{H} \\ -1.67200797572498 & \mathrm{H} \\ 0.00000000000000 & \mathrm{H} \\ 0.00000000000000 & \mathrm{H} \\ 0.00000000000000 & \mathrm{H} \\ 0.00000000000000 & \mathrm{H}\end{array}$
( C C C . C C . , C H H .

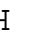

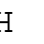

H 


\section{$\mathrm{E}-\left(\mathrm{H}_{2} \mathrm{O}\right)_{2}$ cluster in the ground state $\left(\mathrm{C}_{1}\right.$ symmetry $)$}

1.77749473749690

$-0.56390292550032$

$-0.41260138497745$

1. 86262057611690

4.13591544291979

4. 01454034940696

$-3.00926688186584$

$-5.16517874818103$

$-4.95468688582017$

$-2.56075028958891$

$-7.10968629389566$

6.05174544729884

1. 72423260374984

10.43879982151380

$-6.41232257042878$

$-3.20800193662309$

$-7.04715761126823$

5.83584609742528

$-2.33872252800341$

$-6.78790712352508$

3. 64488813858266

0.73610124064889

0.70447372973498

$-7.18819031438851$

$-6.61121031945744$

9.11980785301915

11.75518747610499
$-3.09802870979528$

$-1.67056105267472$

0.99457261861379

2. 19788833560893

0.92550978991604

- 1.81170692407183

$-2.74749778102166$

$-1.26236600538440$

1. 39967050471638

2. 52722286370797

2. 75268967585890

2.19743463649799

$-5.93745934410338$

$-0.87548898922274$

7.97843251103972

$-4.80149214780550$

$-2.10383212885124$

$-2.77859053743010$

4.57663957171487

4.57107630031087

$-6.71853432509431$

$-6.68221321274101$

$-6.65328181430655$

8.86624551332624

9.05499695477860

0.41157672151343

$-0.16562430711269$
0.01106923161295

0.05056125805012

0.03974600206376

0.01406555669748

$-0.00856720613852$

$-0.01504382591292$

0.08023550626477

0.09509246599698

0.07986475984409

0.05605988325917

0.09074461555460

$-0.02309333743011$

$-0.00936864659634$

$-0.19658724973113$

$-0.13471956339926$

0.08887031968654

0.11769475440890

$-0.05279305109132$

0.03570976178598

0.05428304100722

$-0.03438861814380$

1. 66565789158439

$-1.67868970853597$

$-1.52454084141154$

1. 32245223464732

$-0.13329946044630$

$-1.23171672342531$
C

C

C

O

C

C

C

C

C

O

O

C

$\mathrm{H}$

$\mathrm{H}$

$\mathrm{H}$

$\mathrm{H}$

$\mathrm{H}$

$\mathrm{H}$

$\mathrm{H}$

$\mathrm{H}$

$\mathrm{H}$

$\mathrm{H}$

$\mathrm{H}$

$\mathrm{H}$

$E=-763.51793030147$

\section{$\mathrm{E}-\left(\mathrm{H}_{2} \mathrm{O}\right)_{2}$ cluster in ${ }^{1} \pi \pi^{*}$ state $\left(\mathrm{C}_{\mathrm{s}}\right.$ symmetry $)$}

$-2.50075155472827$

$-0.35759268420573$

$-0.53607658120594$

$-3.01070506473277$

$-5.15713695316931$

$-4.91929408895601$

1. 88659584184036

4. 20550068468672

4.05080553520861

1. 75401182929777

1. 66699397794897

6.09814475908354

$-7.04936264115763$

$-6.49856604660783$

10.56683840748805

$-3.21996399452619$

$-7.05183235729365$

5.86501399905257

$-2.26383525373776$

$-6.72348229315332$
2.47837402721242

0.96290560819086

$-1.77938937483408$

$-2.83212820760749$

$-1.31672561958604$

1. 36765058967505

2. 16608070038832

0.78745588365236

$-1.89556445368566$

$-3.25553033259139$

$-6.07527320194685$

2. 13025057433610

2. 72506827677319

7. 89054268197886

$-0.78438401390152$

$-4.88276079641287$

$-2.13197082932274$

$-2.87827938075707$

4. 52538288348846

4. 55227820358714
0.00000000000000

0.00000000000000

0.00000000000000

0.00000000000000

0.00000000000000

0.00000000000000

0.00000000000000

0.00000000000000

0.00000000000000

0.00000000000000

0.00000000000000

0.00000000000000

0.00000000000000

0.00000000000000

0.00000000000000

0.00000000000000

0.00000000000000

0.00000000000000

0.00000000000000

0.00000000000000
C

C

C

C

C

O

C

C

C

C

O

O

O

O

$\mathrm{H}$

$\mathrm{H}$

$\mathrm{H}$

$\mathrm{H}$

$\mathrm{H}$ 
3.58313913716248

0.66410423495763

0.66410423495763

$-5.04306884431293$

$-7.96139529122011$

9. 17152880528036

12.07808704125046
$-6.87294081326808$

$-6.83654216641885$

$-6.83654216641885$

8.98549038990582

8.97590976999690

0.42292291659298

0.22604601912873
0.00000000000000

1. 67215024420880

$-1.67215024420880$

0.00000000000000

0.00000000000000

0.00000000000000

0.00000000000000
$\mathrm{H}$

$\mathrm{H}$

$\mathrm{H}$

$\mathrm{H}$

$\mathrm{H}$

$\mathrm{H}$

$\mathrm{H}$

$$
E=-763.3741201115129
$$

\section{E- $\left(\mathrm{H}_{2} \mathrm{O}\right)_{2}$ cluster in ${ }^{1} \pi \pi^{*}$ state $\left(\mathrm{C}_{1}\right.$ symmetry $)$}

\begin{abstract}
1. 72887380022596
$-0.56365534163231$

$-0.39280693051330$

1. 85137617786654

4. 17194980408097

4.02333670603796

$-3.03618450972045$

$-5.18362540886571$

$-4.95106884901975$

$-2.53717454024100$

$-7.09091614778474$

6.06440309956448

1. 65294639497973

10.53019969420086

$-6.35739647545624$

$-3.24563445798962$

$-7.07756628285393$

5.83956801891483

$-2.31319652208944$

$-6.77377254144087$

3. 57365323596524

0.67544528910957

0.63589413276695

$-6.84551232414036$

$-6.75962687169590$

9.14482963506073

11.80329459636362
\end{abstract}

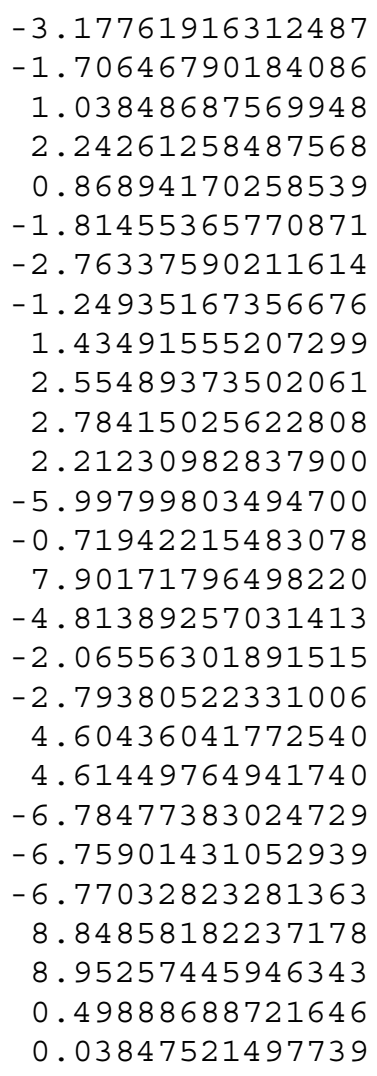

0.00096223386310

0.02976117727865

0.01170172022319

$-0.02602666099408$

$-0.04841644507509$

$-0.03953619457712$

0.07270632618989

0.09215561105840

0.06875066159504

0.03237741256772

0.07959314048704

$-0.07462839870926$

0.01135894863059

$-0.17954589692100$

$-0.05849474310876$

0.08946508984227

0.12226996119474

$-0.07045845577884$

0.01709684861521

0.04478213135111

$-0.00830922985358$

1. 69868994541969

$-1.64721020198468$

$-1.53792784223750$

1. 37579650423917

$-0.13901684797746$

$-1.23405347015971$
C
C
C
O
C
C
C
C
C
C
$\mathrm{O}$
$\mathrm{O}$
$\mathrm{C}$
$\mathrm{O}$
$\mathrm{O}$
$\mathrm{H}$
$\mathrm{H}$
$\mathrm{H}$
$\mathrm{H}$
$\mathrm{H}$
$\mathrm{H}$
$\mathrm{H}$
$\mathrm{H}$
$\mathrm{H}$
$\mathrm{H}$
$\mathrm{H}$
$\mathrm{H}$

$E=-763.3748729815624$

\section{$\mathrm{E}-\left(\mathrm{H}_{2} \mathrm{O}\right)_{2}$ cluster in ${ }^{1} n \pi^{*}$ state $\left(\mathrm{C}_{\mathrm{s}}\right.$ symmetry $)$}

-2.47856777365111
-0.38772116020788
-0.55362071893145
-3.03747904730762
-5.13625033313926
-5.00454742506043
1.89553969654934
4.15001676810328
3.99768345568827
1.78360757127729
1.74522321524582
2.45607200339126

0.96620494811616

- 1.74555446438708

$-2.82804522355116$

$-1.39571025883259$

1. 32137412333729

2.13466913163228

0.88979996557251

$-1.86095908004206$

$-3.13489832870456$

$-5.98864884511858$
0.00000000000000

0.00000000000000

0.00000000000000

0.00000000000000

0.00000000000000

0.00000000000000

0.00000000000000

0.00000000000000

0.00000000000000

0.00000000000000

0.00000000000000
C

$\mathrm{C}$

C

C

C

C

O

C

C

C

C 
6.06035750301481

$-6.95004663118808$

$-6.72580033716363$

10.44783403371540

$-3.22394326620430$

$-7.07652947436141$

5.84787873942403

$-2.22488641157851$

$-6.99346376789302$

3. 66886921003706

0.77317594151706

0.77317594151706

$-4.85614339650838$

$-7.93806138483457$

9. 23961007680868

12.07165931461705

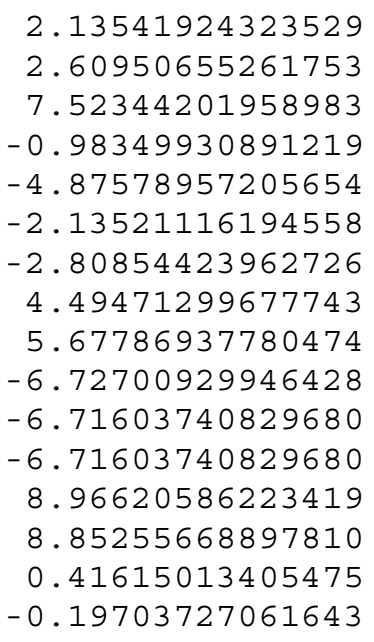

2.13541924323529

0.00000000000000

0.00000000000000

0.00000000000000

0.00000000000000

0.00000000000000

0.00000000000000

0.00000000000000

0.00000000000000

0.00000000000000

0.00000000000000

1. 67936155166059

$-1.67936155166059$

0.00000000000000

0.00000000000000

0.00000000000000

0.00000000000000
0

0

O

$\mathrm{O}$

$\mathrm{H}$

$\mathrm{H}$

$\mathrm{H}$

$\mathrm{H}$

$\mathrm{H}$

$\mathrm{H}$

$\mathrm{H}$

$\mathrm{H}$

$\mathrm{H}$

$\mathrm{H}$

$\mathrm{H}$

$\mathrm{H}$

$E=-763.3816337575419$

\section{$\mathrm{E}-\left(\mathrm{H}_{2} \mathrm{O}\right)_{4}$ cluster in the ground state $\left(\mathrm{C}_{\mathrm{s}}\right.$ symmetry $)$}

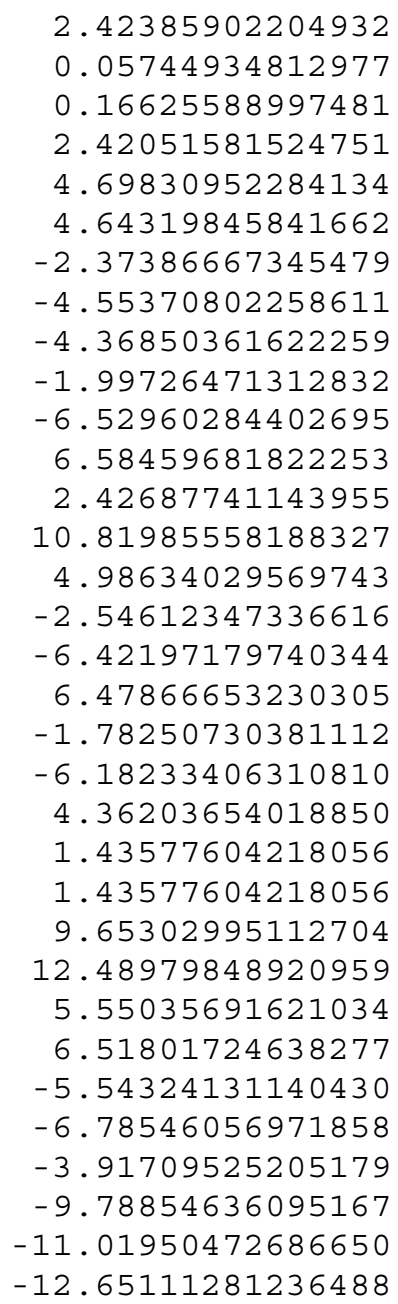

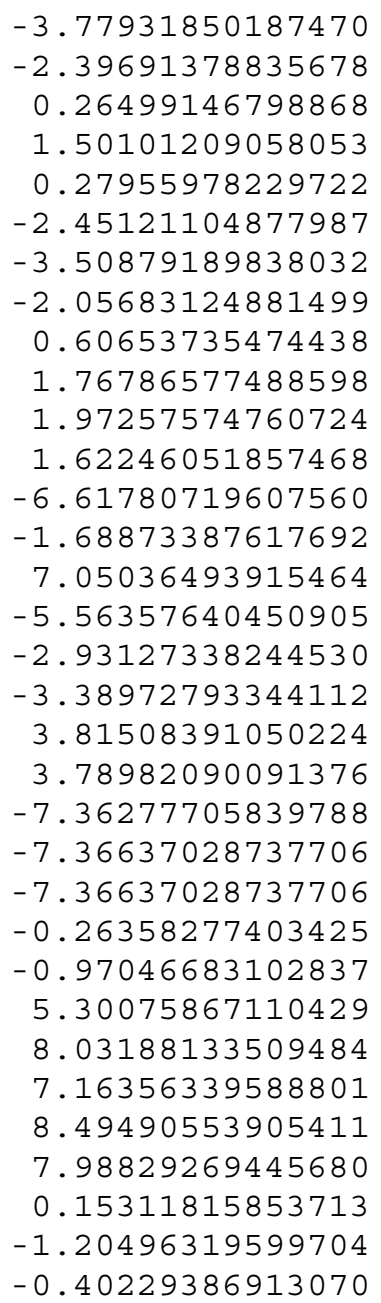

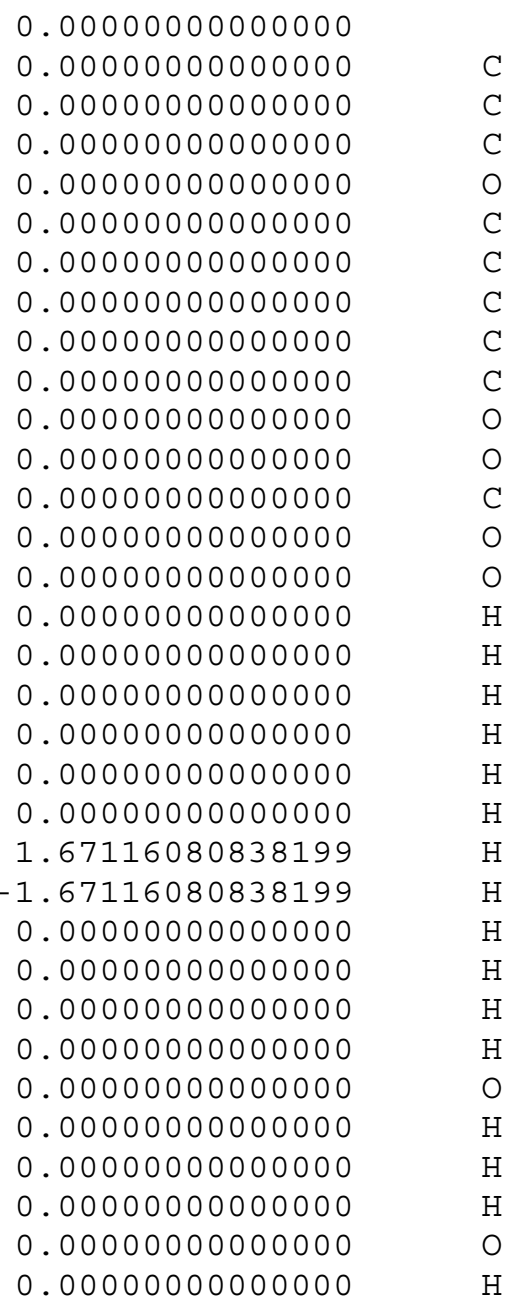

$E=-916.22007070123$ 


\section{$\mathrm{E}-\left(\mathrm{H}_{2} \mathrm{O}\right)_{4}$ cluster in ${ }^{1} \pi \pi^{*}$ excited state $\left(\mathrm{C}_{\mathrm{s}}\right.$ symmetry $)$}

2.34344040884507

0.02525250400538

0.17098900681909

2.40273802832795

4. 71348125684684

4.61893214867941

$-2.44017168159013$

$-4.60588878857316$

$-4.37945532903856$

$-1.97442321462460$

$-6.50738390423169$

6.58756581481562

2. 30095826422966

11.03466105562986

5.04617902564919

$-2.63432320850448$

$-6.49268556675915$

6.44813471029799

$-1.73777206124001$

$-6.15616614129101$

4. 22852896886408

1. 30910424928295

1. 30910424928295

9. 67693458009420

12.57942735773726

5.54573430289346

6.61555885596925

$-5.69274174966942$

$-7.01436185171200$

$-4.11568301475698$

$-9.89188559766213$

$-10.96775347935787$

$-12.67716938600475$

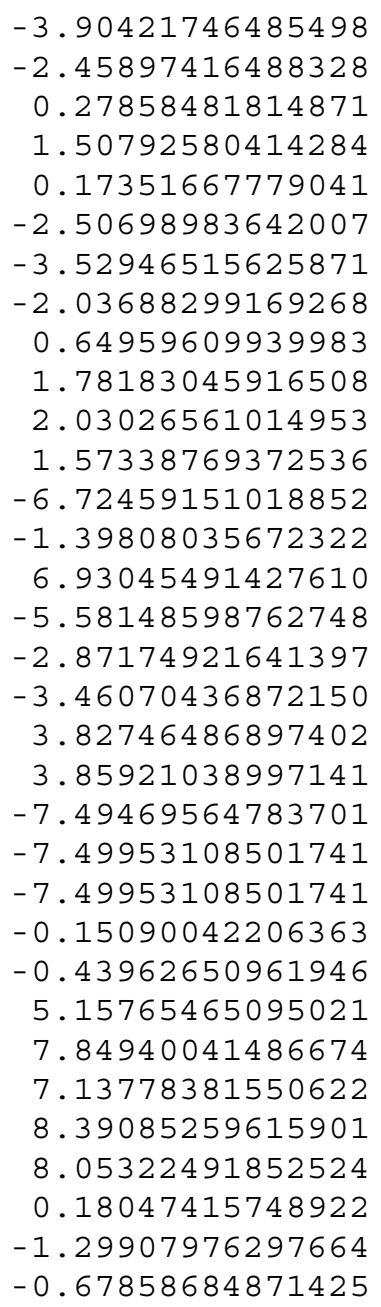

0.00000000000000

0.00000000000000

0.00000000000000

0.00000000000000

0.00000000000000

0.00000000000000

0.00000000000000

0.00000000000000

0.00000000000000

0.00000000000000

0.00000000000000

0.00000000000000

0.00000000000000

0.00000000000000

0.00000000000000

0.00000000000000

0.00000000000000

0.00000000000000

0.00000000000000

0.00000000000000

0.00000000000000

1. 67195357998990

$-1.67195357998990$

0.00000000000000

0.00000000000000

0.00000000000000

0.00000000000000

0.00000000000000

0.00000000000000

0.00000000000000

0.00000000000000

0.00000000000000

0.00000000000000
C

C

C

O

C

C

C

C

C

C

O

C

O

$\mathrm{H}$

$\mathrm{H}$

$\mathrm{H}$

$\mathrm{H}$

$\mathrm{H}$

$\mathrm{H}$

$\mathrm{H}$

$\mathrm{H}$

$\mathrm{H}$

$\mathrm{H}$

$\mathrm{H}$

O

$\mathrm{H}$

$\mathrm{H}$

O

$\mathrm{H}$

$E=-916.0773247609771$

\section{$\mathbf{E}-\left(\mathrm{H}_{2} \mathrm{O}\right)_{4}$ cluster in ${ }^{1} n \pi^{*}$ excited state $\left(\mathrm{C}_{\mathrm{s}}\right.$ symmetry)}

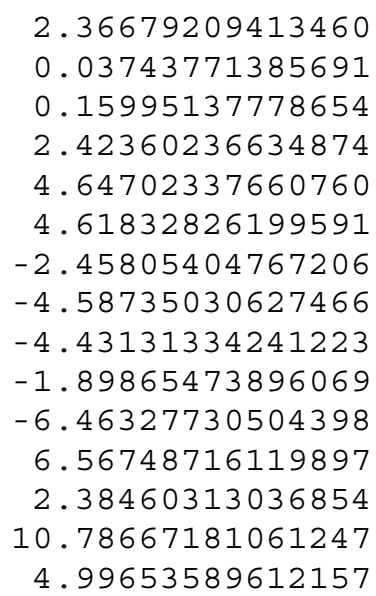

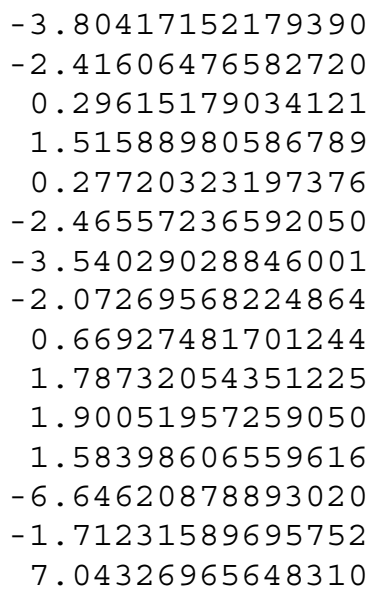

0.00000000000000

0.00000000000000

0.00000000000000

0.00000000000000

0.00000000000000

0.00000000000000

0.00000000000000

0.00000000000000

0.00000000000000

0.00000000000000

0.00000000000000

0.00000000000000

0.00000000000000

0.00000000000000

0.00000000000000
C

C

C

O

C

C

C

C

C

C

O

O

C

O 
$-2.58420183201974$

$-6.46635351646608$

6.44786032044513

$-1.74311102726273$

$-5.99155292569669$

4. 33902209715496

1. 41636897240075

1. 41636897240075

9. 67070030256409

12.49260920519760

5.59375701418660

6.46233908486582

$-5.38331107870382$

$-7.26258044462512$

$-4.01145186564735$

$-9.85707590894098$

$-11.01836434960614$

$-12.69539789098061$
$-5.58781960854131$

$-2.94337799961015$

$-3.40595225282066$

3. 81283079446086

4.60172267202173

$-7.37477722476968$

$-7.40797696174930$

$-7.40797696174930$

$-0.26114870210735$

$-1.03677511247853$

5. 29063975842329

8.03169300431228

6.52163831155185

8. 37845998554977

7. 58897836459809

0.15317899811623

$-1.22375148661076$

- 0.48931230151382
0.00000000000000

0.00000000000000

0.00000000000000

0.00000000000000

0.00000000000000

0.00000000000000

1. 67470112072788

$-1.67470112072788$

0.00000000000000

0.00000000000000

0.00000000000000

0.00000000000000

0.00000000000000

0.00000000000000

0.00000000000000

0.00000000000000

0.00000000000000

0.00000000000000
$\mathrm{H}$

$\mathrm{H}$

$\mathrm{H}$

$\mathrm{H}$

$\mathrm{H}$

$\mathrm{H}$

$\mathrm{H}$

$\mathrm{H}$

$\mathrm{H}$

$\mathrm{H}$

$\mathrm{H}$

$\mathrm{H}$

O

$\mathrm{H}$

$\mathrm{H}$

$\mathrm{H}$

O

$\mathrm{H}$

$E=-916.0810078032321$

\section{$\mathrm{K}-\left(\mathrm{H}_{2} \mathrm{O}\right)_{2}$ cluster in the ground $\operatorname{state}\left(\mathrm{C}_{\mathrm{s}}\right.$ symmetry $)$}

2.47809729269515

0.19604578190235

0.24320710546291

2. 57471706341197

4. 73744594576614

4.78444205526422

$-2.27512918813525$

$-4.42726176800556$

$-4.36642409649444$

$-1.87172216810100$

$-6.33814514816715$

6.77676770204296

2. 54669708656196

$-2.41624579857066$

$-6.28980736016783$

6.61117152762212

$-1.73236225611609$

4.49747999139864

1.57782882134484

1. 57782882134484

6.37512934902657

$-9.29280845681275$

$-10.71194042460669$

$-12.21483895688310$

5. 77473796893518

4.05035030273086

6.84734376575758

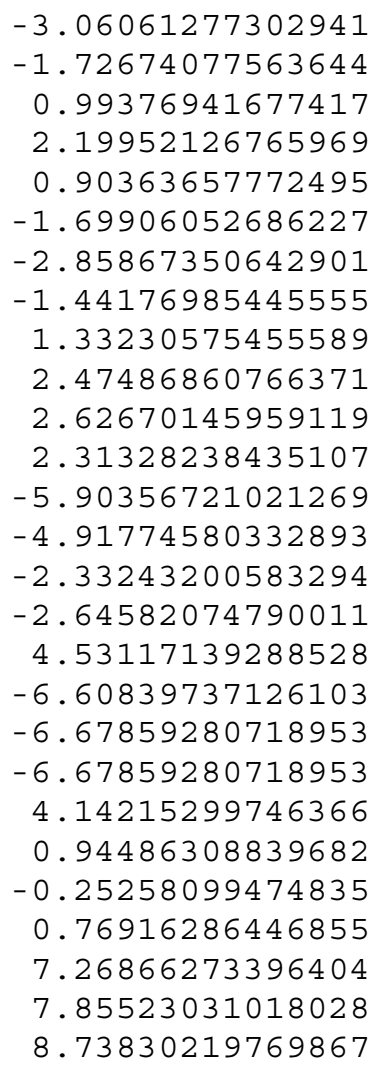

$\begin{array}{ll}0.00000000000000 & \mathrm{C} \\ 0.00000000000000 & \mathrm{C} \\ 0.00000000000000 & \mathrm{C} \\ 0.00000000000000 & \mathrm{O} \\ 0.00000000000000 & \mathrm{C} \\ 0.00000000000000 & \mathrm{C} \\ 0.00000000000000 & \mathrm{C} \\ 0.00000000000000 & \mathrm{C} \\ 0.00000000000000 & \mathrm{C} \\ 0.00000000000000 & \mathrm{C} \\ 0.00000000000000 & \mathrm{O} \\ 0.00000000000000 & \mathrm{O} \\ 0.00000000000000 & \mathrm{C} \\ 0.00000000000000 & \mathrm{H} \\ 0.00000000000000 & \mathrm{H} \\ 0.00000000000000 & \mathrm{H} \\ 0.00000000000000 & \mathrm{H} \\ 0.00000000000000 & \mathrm{H} \\ 1.67141418144063 & \mathrm{H} \\ -1.67141418144063 & \mathrm{H} \\ 0.00000000000000 & \mathrm{H} \\ 0.00000000000000 & \mathrm{H} \\ 0.00000000000000 & \mathrm{O} \\ 0.00000000000000 & \mathrm{H} \\ 0.00000000000000 & \mathrm{O} \\ 0.00000000000000 & \mathrm{H} \\ 0.00000000000000 & \mathrm{H}\end{array}$

C

C

C

$\mathbf{E}=-763.48477943019$

C

C

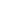

(n) (1) (n) H H H (n) (n) H (n) (1)

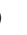

H

(1)

\section{$\mathrm{K}-\left(\mathrm{H}_{2} \mathrm{O}\right)_{2}$ cluster in ${ }^{1} \pi \pi^{*}$ state $\left(\mathrm{C}_{\mathrm{s}}\right.$ symmetry $)$}




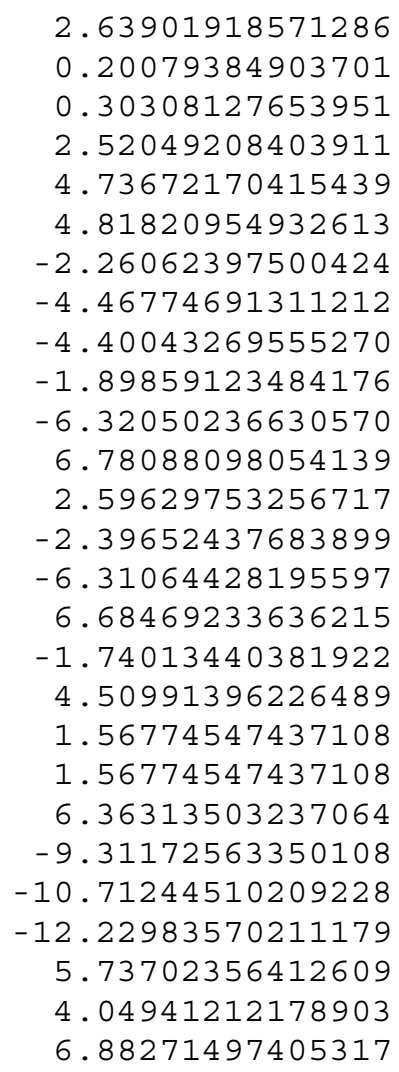

$\begin{array}{ll}0.00000000000000 & \mathrm{C} \\ 0.00000000000000 & \mathrm{C} \\ 0.00000000000000 & \mathrm{C} \\ 0.00000000000000 & \mathrm{O} \\ 0.00000000000000 & \mathrm{C} \\ 0.00000000000000 & \mathrm{C} \\ 0.00000000000000 & \mathrm{C} \\ 0.00000000000000 & \mathrm{C} \\ 0.00000000000000 & \mathrm{C} \\ 0.00000000000000 & \mathrm{C} \\ 0.00000000000000 & \mathrm{O} \\ 0.00000000000000 & \mathrm{O} \\ 0.00000000000000 & \mathrm{C} \\ 0.00000000000000 & \mathrm{H} \\ 0.00000000000000 & \mathrm{H} \\ 0.00000000000000 & \mathrm{H} \\ 0.00000000000000 & \mathrm{H} \\ 0.00000000000000 & \mathrm{H} \\ 1.66476239604312 & \mathrm{H} \\ -1.66476239604312 & \mathrm{H} \\ 0.00000000000000 & \mathrm{H} \\ 0.00000000000000 & \mathrm{H} \\ 0.00000000000000 & \mathrm{O} \\ 0.00000000000000 & \mathrm{H} \\ 0.00000000000000 & \mathrm{O} \\ 0.00000000000000 & \mathrm{H} \\ 0.00000000000000 & \mathrm{H}\end{array}$

0.00000000000000

C

$-1.74586938267480$

0.87794100647181

2. 16954383084721

0.90708450706508

$-1.76358961017871$

$-2.84937219832747$

$-1.45990087024029$

1. 27102930424088

2. 41150392253436

2. 67971798622048

2. 34759772652474

$-5.97909082321801$

$-4.91048816950308$

$-2.38419849055270$

$-2.63995137728472$

4.46635831160105

$-6.78186830048899$

$-6.71878422684517$

$-6.71878422684517$

4. 16415675103797

0.98457642520454

$-0.22926777843796$

0.77023151075898

7. 36826243663098

8.05232234130542

8.78245556784062

C

O

C

C

$\mathrm{C}$

C

O H H $\mathrm{H}$ $\mathrm{H}$ $\mathrm{H}$ $\mathrm{H}$ $\mathrm{H}$ (1)

$\mathrm{H}$ $\mathrm{H}$ $\mathrm{H}$ H $\mathrm{H}$

\section{$\mathbf{K}-\left(\mathrm{H}_{2} \mathrm{O}\right)_{2}$ cluster in ${ }^{1} \mathrm{n} \pi^{*}$ state $\left(\mathrm{C}_{\mathrm{s}}\right.$ symmetry $)$}

\footnotetext{
2.61705764132251

0.25163404664465

0.32088809814546

2.59338870677406

4.79766548439223

4.85372778590373

$-2.17977758464713$

$-4.38665332637895$

$-4.22917429371568$

$-1.83881367385046$

$-6.30617545372389$

6.83751107205551

2.62784937151849

$-2.36692671594007$

$-6.24570200525519$

6.70703922330614

$-1.66842093607118$

4.56502633638853

1.64139962225655

1.64139962225655

6.40854252141300

- 9.84389947266645

$-11.02324044067623$
}
$-3.14658296430489$
$-1.74883185828512$
0.91008194832192
2. 19058930978495
0.87994388373461
$-1.72851405645209$
$-2.83891617530600$
$-1.39884462152458$
1. 26513258118355
2. 42510301980952
2. 65507541115761
2. 32912266051134
$-5.96942230514810$
$-4.89429864763849$
$-2.28714127025183$
$-2.63072783645389$
4.47595127527391
$-6.71704385779566$
$-6.75043251790895$
$-6.75043251790895$
4.13935425650959
1. 24043051007667
$-0.15885645406239$

$\begin{array}{cc}0.00000000000000 & \mathrm{C} \\ 0.00000000000000 & \mathrm{C} \\ 0.00000000000000 & \mathrm{C} \\ 0.00000000000000 & \mathrm{O} \\ 0.00000000000000 & \mathrm{C} \\ 0.00000000000000 & \mathrm{C} \\ 0.00000000000000 & \mathrm{C} \\ 0.00000000000000 & \mathrm{C} \\ 0.00000000000000 & \mathrm{C} \\ 0.00000000000000 & \mathrm{C} \\ 0.00000000000000 & \mathrm{O} \\ 0.00000000000000 & \mathrm{O} \\ 0.00000000000000 & \mathrm{C} \\ 0.00000000000000 & \mathrm{H} \\ 0.00000000000000 & \mathrm{H} \\ 0.00000000000000 & \mathrm{H} \\ 0.00000000000000 & \mathrm{H} \\ 0.00000000000000 & \mathrm{H} \\ 1.66908762598510 & \mathrm{H} \\ -1.66908762598510 & \mathrm{H} \\ 0.00000000000000 & \mathrm{H} \\ 0.00000000000000 & \mathrm{H} \\ 0.00000000000000 & \mathrm{O}\end{array}$ 
-12.67266886810295
5.78612138334629
4.06182948176496
6.85099954256806

$E=-763.3810305896133$
0.60759709720240

7.35515838114203

7.93873851383362

8.83016748327823
0.00000000000000

0.00000000000000

0.00000000000000

0.00000000000000
$\mathrm{H}$

O

$\mathrm{H}$

$\mathrm{H}$

\section{$\mathrm{K}-\left(\mathrm{H}_{2} \mathrm{O}\right)_{4}$ cluster in the ground state $\left(\mathrm{C}_{\mathrm{s}}\right.$ symmetry $)$}

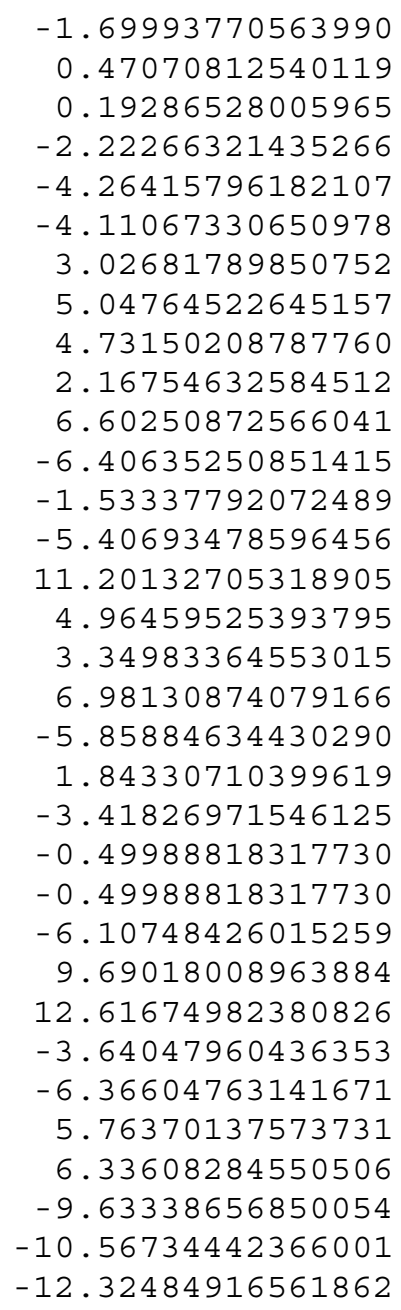

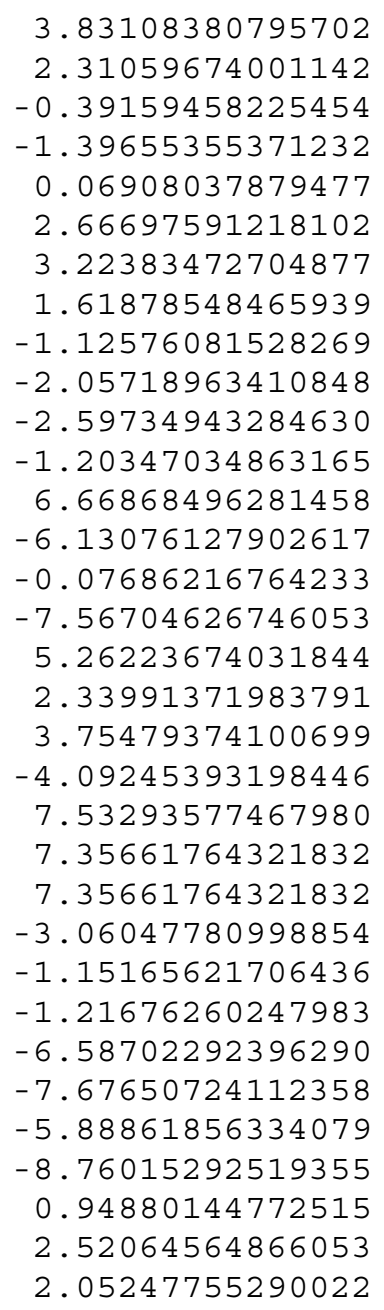

$\begin{array}{ll}0.00000000000000 & \mathrm{C} \\ 0.0000000000000 & \mathrm{C} \\ 0.0000000000000 & \mathrm{C} \\ 0.00000000000000 & \mathrm{O} \\ 0.00000000000000 & \mathrm{C} \\ 0.0000000000000 & \mathrm{C} \\ 0.00000000000000 & \mathrm{C} \\ 0.00000000000000 & \mathrm{C} \\ 0.00000000000000 & \mathrm{C} \\ 0.0000000000000 & \mathrm{C} \\ 0.00000000000000 & \mathrm{O} \\ 0.00000000000000 & \mathrm{O} \\ 0.00000000000000 & \mathrm{C} \\ 0.0000000000000 & \mathrm{O} \\ 0.00000000000000 & \mathrm{O} \\ 0.00000000000000 & \mathrm{O} \\ 0.0000000000000 & \mathrm{H} \\ 0.00000000000000 & \mathrm{H} \\ 0.00000000000000 & \mathrm{H} \\ 0.00000000000000 & \mathrm{H} \\ 0.0000000000000 & \mathrm{H} \\ 1.67101094728486 & \mathrm{H} \\ -1.67101094728486 & \mathrm{H} \\ 0.00000000000000 & \mathrm{H} \\ 0.00000000000000 & \mathrm{H} \\ 0.00000000000000 & \mathrm{H} \\ 0.00000000000000 & \mathrm{H} \\ 0.00000000000000 & \mathrm{H} \\ 0.00000000000000 & \mathrm{H} \\ 0.00000000000000 & \mathrm{H} \\ 0.00000000000000 & \mathrm{H} \\ 0.00000000000000 & \mathrm{O} \\ 0.00000000000000 & \mathrm{H}\end{array}$

C

C

C

C

C

C

C

C

O

O

C

0

$\mathrm{H}$

$\mathrm{H}$

$\mathrm{H}$

$\mathrm{H}$

$\mathrm{H}$

H

$\mathrm{H}$

$\mathrm{H}$

$\mathrm{H}$

$\mathrm{H}$ $\mathrm{H}$ $\mathrm{H}$ $\mathrm{H}$ $\mathrm{H}$ $\mathrm{H}$

$\mathbf{E}=-916.19166425757$

\section{$\mathrm{K}-\left(\mathrm{H}_{2} \mathrm{O}\right)_{4}$ cluster in ${ }^{1} \pi \pi^{*}$ excited state $\left(\mathrm{C}_{\mathrm{s}}\right.$ symmetry $)$}

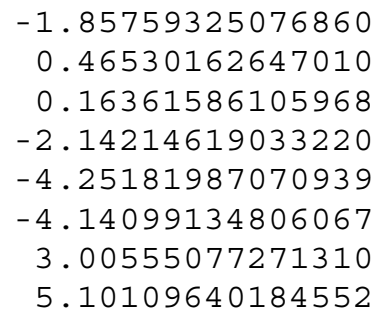

\author{
3.97682070052164 \\ 2. 36645017540149 \\ $-0.23531921480242$ \\ $-1.35671818332607$ \\ 0.07609243874364 \\ 2. 74097025692015 \\ 3. 26757027699829 \\ 1. 70552788990587
}

$\begin{array}{ll}0.00000000000000 & C \\ 0.00000000000000 & C \\ 0.00000000000000 & C \\ 0.00000000000000 & \text { O } \\ 0.00000000000000 & C \\ 0.00000000000000 & C \\ 0.00000000000000 & C \\ 0.00000000000000 & C\end{array}$ 


$$
\begin{array}{r}
4.81386145501341 \\
2.23802115023597 \\
6.63234371469983 \\
-6.39325941255117 \\
-1.60242596178994 \\
-5.39526241823339 \\
11.29180730461205 \\
4.75568429921683 \\
3.30676560206749 \\
7.00841540285941 \\
-5.93621196133585 \\
1.90612555136722 \\
-3.45092520001200 \\
-0.52374229906971 \\
-0.52374229906971 \\
-6.07477612335194 \\
9.78232614984214 \\
12.70694697295975 \\
-3.64905563982295 \\
-6.40536464152739 \\
5.69806346675094 \\
6.00927460493016 \\
-9.59603498047113 \\
-10.43964384457983 \\
-12.22239510065974
\end{array}
$$

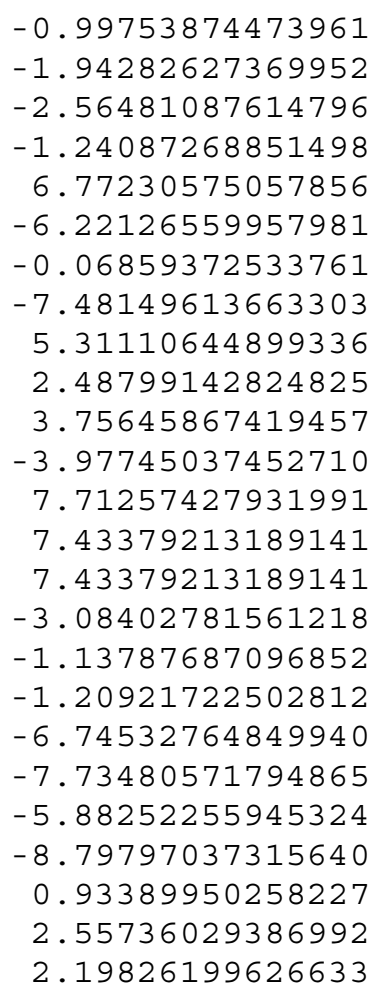
$\begin{array}{ll}0.00000000000000 & \mathrm{C} \\ 0.00000000000000 & \mathrm{C} \\ 0.00000000000000 & \mathrm{O} \\ 0.00000000000000 & \mathrm{O} \\ 0.00000000000000 & \mathrm{C} \\ 0.00000000000000 & \mathrm{O} \\ 0.00000000000000 & \mathrm{O} \\ 0.00000000000000 & \mathrm{O} \\ 0.00000000000000 & \mathrm{H} \\ 0.00000000000000 & \mathrm{H} \\ 0.00000000000000 & \mathrm{H} \\ 0.00000000000000 & \mathrm{H} \\ 0.0000000000000 & \mathrm{H} \\ 1.66616646554833 & \mathrm{H} \\ -1.66616646554833 & \mathrm{H} \\ 0.00000000000000 & \mathrm{H} \\ 0.00000000000000 & \mathrm{H} \\ 0.00000000000000 & \mathrm{H} \\ 0.00000000000000 & \mathrm{H} \\ 0.00000000000000 & \mathrm{H} \\ 0.00000000000000 & \mathrm{H} \\ 0.00000000000000 & \mathrm{H} \\ 0.00000000000000 & \mathrm{H} \\ 0.00000000000000 & \mathrm{O} \\ 0.00000000000000 & \mathrm{H}\end{array}$

$$
E=-916.0902697572414
$$
C , , ,

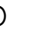$$
\text { O }
$$

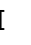

\section{.}$$
\text { . }
$$

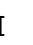

$$
\text { H }
$$
H H H

H

$\mathrm{H}$

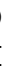




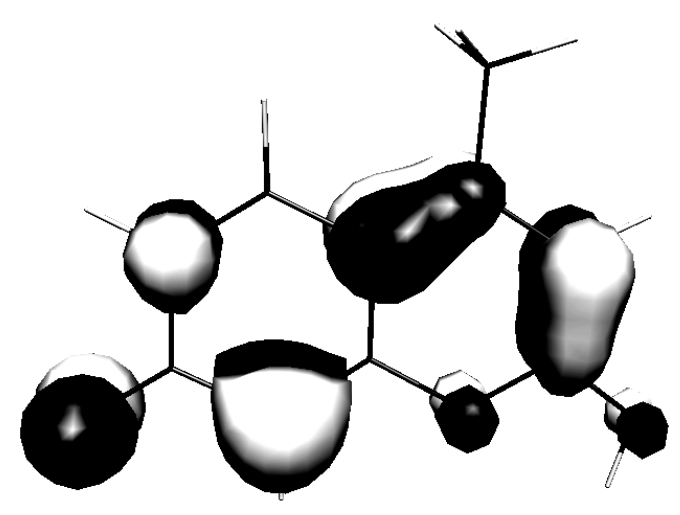

HOMO

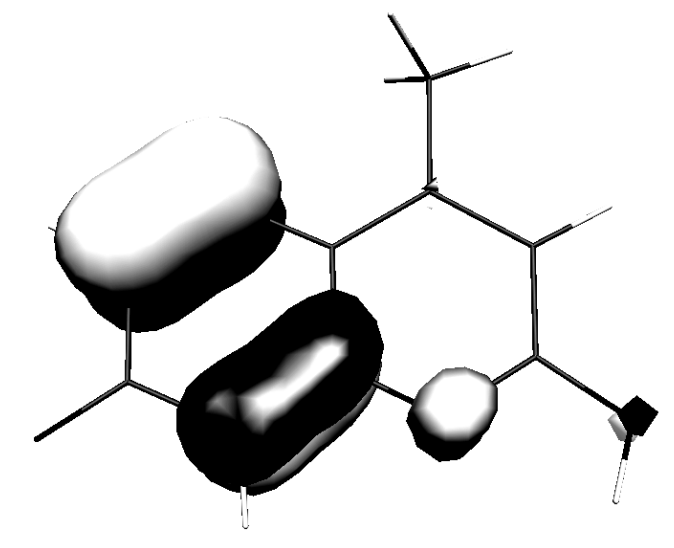

HOMO-2

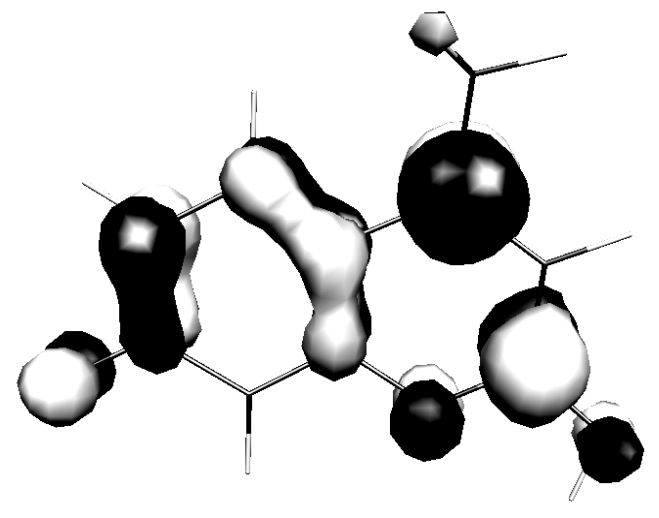

LUMO

Figure 3a. LUMO $\left(\pi^{*}\right)$, HOMO $(\pi)$ and HOMO-2 (n) orbitals of keto form. 


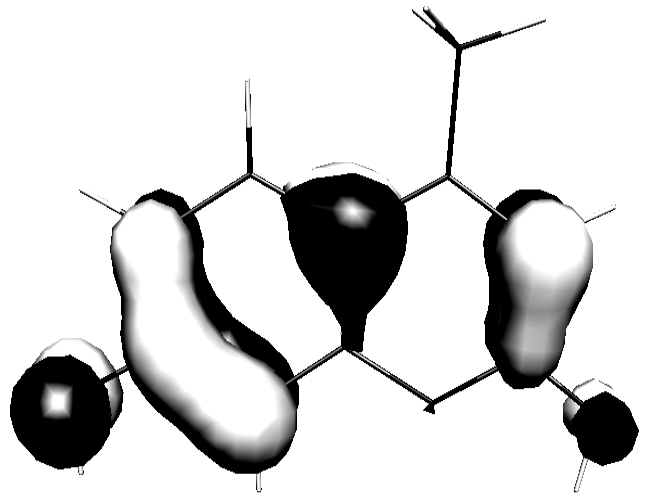

HOMO

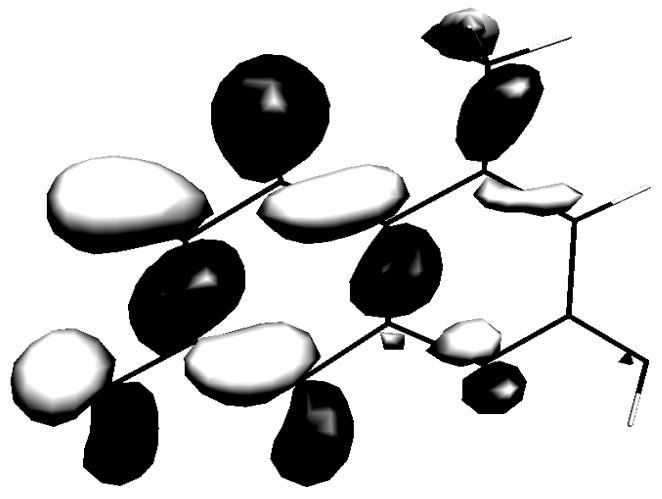

HOMO-4

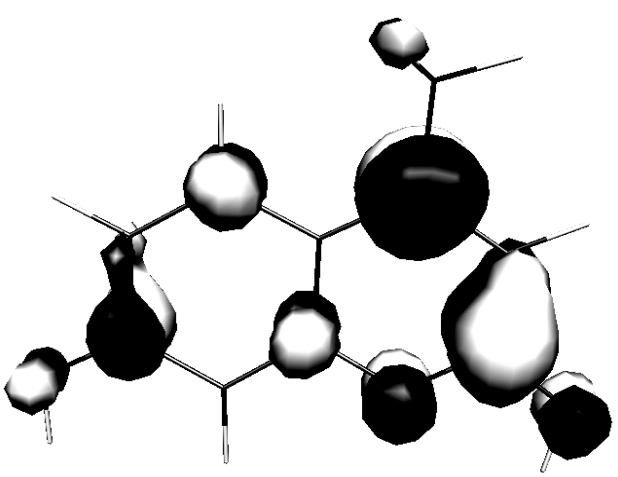

LUMO

Figure $3 b$. LUMO $\left(\pi^{*}\right)$, HOMO $(\pi)$ and HOMO-4 (n) orbitals of cationic form. 


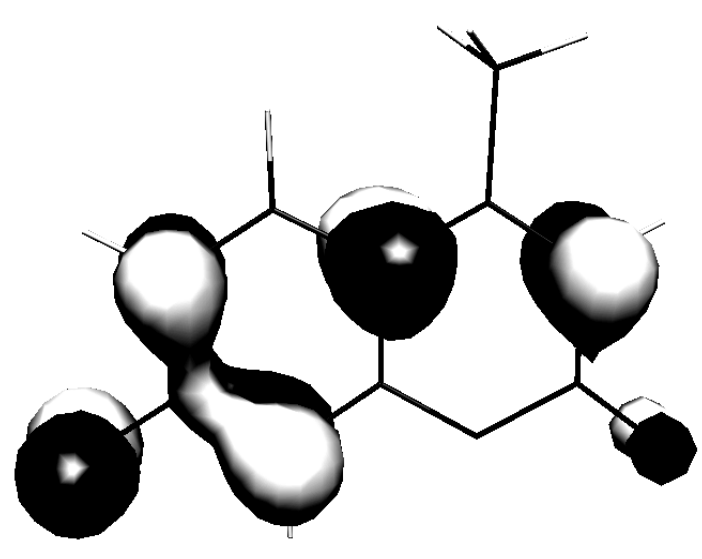

HOMO

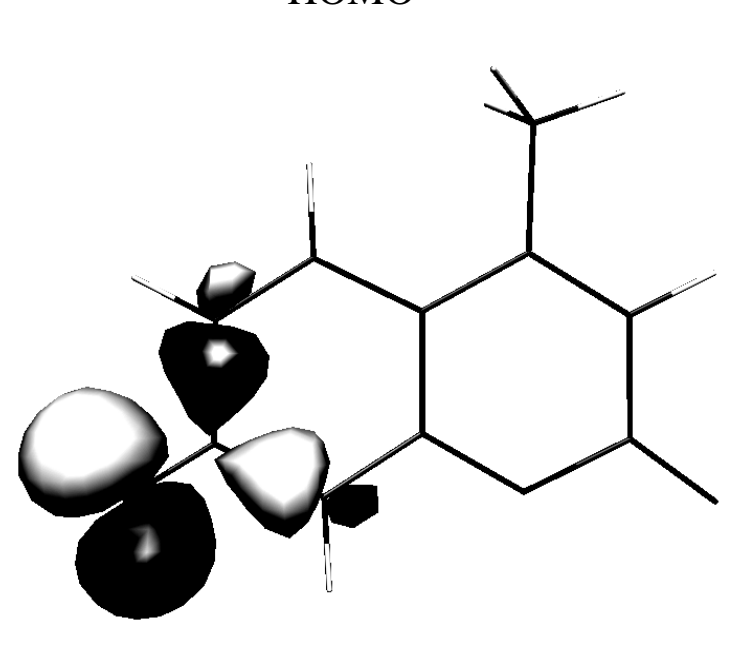

HOMO-1

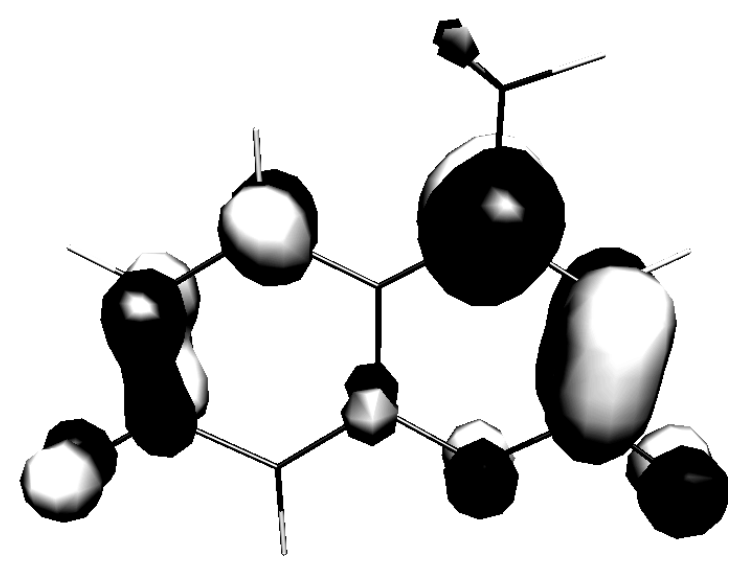

LUMO

-

Figure 3c. LUMO $\left(\pi^{*}\right)$, HOMO $(\pi)$ and HOMO-1 (n) orbitals of anionic form. 
TABLE 5a: TDDFT/B3LYP minimum-to-minimum (m.-m.) and zero-point corrected $(0-0)$ absorption energies $(\mathrm{eV})$ as well as vertical fluorescence transitions (fl.) for the $E$, $\mathrm{K}, \mathrm{C}$ and $\mathrm{A}$ forms of $7 \mathrm{H} 4 \mathrm{MC}$

\begin{tabular}{|c|c|c|c|c|}
\hline Geometry & State & SVP & TZVP & $\operatorname{SVPD}^{a}$ \\
\hline & enol $(\mathbf{E})$ & & & \\
\hline \multirow[t]{3}{*}{$\pi \pi *\left(\mathrm{C}_{\mathrm{s}}\right)$} & m.-m. $2^{1} A^{\prime}\left(\pi \pi^{*}\right)$ & 3.98 & 3.96 & 3.93 \\
\hline & $0-0\left(\pi \pi^{*}\right)$ & - & - & 3.78 \\
\hline & fl. $2^{1} \mathrm{~A}^{\prime}\left(\pi \pi^{*}\right)$ & 3.76 & 3.70 & 3.73 \\
\hline \multirow[t]{2}{*}{$\mathrm{n} \pi^{*}\left(C_{\mathrm{s}}\right)$} & m.-m. $1^{1} \mathrm{~A}^{\prime \prime}\left(\mathrm{n} \pi^{*}\right)$ & 3.81 & 3.87 & 3.86 \\
\hline & $\begin{array}{l}\text { fl. } 1^{1} \mathrm{~A}^{\prime \prime}\left(\mathrm{n} \pi^{*}\right) \\
\text { keto }(\mathbf{K})\end{array}$ & 3.00 & 3.06 & 3.08 \\
\hline \multirow[t]{3}{*}{$\pi \pi *\left(\mathrm{C}_{\mathrm{s}}\right)$} & m.-m. $2^{1} \mathrm{~A}^{\prime}\left(\pi \pi^{*}\right)$ & 2.74 & 2.75 & 2.70 \\
\hline & $0-0\left(\pi \pi^{*}\right)$ & - & - & 2.63 \\
\hline & fl. $2^{1} \mathrm{~A}^{\prime}\left(\pi \pi^{*}\right)$ & 2.30 & 2.31 & 2.28 \\
\hline \multirow[t]{2}{*}{$\mathrm{n} \pi^{*}\left(C_{\mathrm{s}}\right)$} & m.-m. $1^{1} \mathrm{~A}^{\prime \prime}\left(\mathrm{n} \pi^{*}\right)$ & 2.43 & 2.52 & 2.50 \\
\hline & $\begin{array}{l}\text { fl. } 1^{1} \mathrm{~A}^{\prime \prime}\left(\mathrm{n} \pi^{*}\right) \\
\text { cation }(\mathbf{C})\end{array}$ & 2.03 & 2.11 & 2.12 \\
\hline \multirow[t]{3}{*}{$\pi \pi^{*}\left(\mathrm{C}_{\mathrm{s}}\right)$} & m.-m. $2^{1} \mathrm{~A}^{\prime}\left(\pi \pi^{*}\right)$ & 3.40 & 3.40 & 3.36 \\
\hline & $0-0\left(\pi \pi^{*}\right)$ & - & - & 3.24 \\
\hline & fl. $2^{1} \mathrm{~A}^{\prime}\left(\pi \pi^{*}\right)$ & 3.05 & 3.04 & 3.02 \\
\hline \multirow[t]{2}{*}{$\mathrm{n} \pi^{*}\left(C_{\mathrm{s}}\right)$} & m.-m. $1^{1} \mathrm{~A}^{\prime \prime}\left(\mathrm{n} \pi^{*}\right)$ & 5.76 & 5.76 & 5.70 \\
\hline & $\begin{array}{l}\text { fl. } 1^{1} \mathrm{~A}^{\prime \prime}\left(\mathrm{n} \pi^{*}\right) \\
\text { anion }(\mathbf{A})\end{array}$ & 4.86 & 4.94 & 4.79 \\
\hline \multirow[t]{3}{*}{$\pi \pi^{*}\left(\mathrm{C}_{\mathrm{s}}\right)$} & m.-m. $2^{1} \mathrm{~A}^{\prime}\left(\pi \pi^{*}\right)$ & 3.05 & 3.04 & 3.00 \\
\hline & $0-0\left(\pi \pi^{*}\right)$ & - & - & 2.97 \\
\hline & fl. $2^{1} \mathrm{~A}^{\prime}\left(\pi \pi^{*}\right)$ & 2.73 & 2.75 & 2.74 \\
\hline \multirow[t]{2}{*}{$\mathrm{n} \pi^{*}\left(C_{\mathrm{s}}\right)$} & m.-m. $1^{1} \mathrm{~A}^{\prime \prime}\left(\mathrm{n} \pi^{*}\right)$ & 2.97 & 3.07 & $3.01\left(\pi \sigma^{*}\right)$ \\
\hline & fl. $1^{1} \mathrm{~A}^{\prime \prime}\left(\mathrm{n} \pi^{*}\right)$ & 2.52 & 2.62 & $2.93\left(\pi \sigma^{*}\right)$ \\
\hline
\end{tabular}

${ }^{a}$ SVPD results are given for comparison (they are included in Table 5). 